\section{Rendezvous technique: double-balloon endoscopy and SpyGlass direct visualization system in a patient with severe stenosis of a choledochojejunal anastomosis}

This is the first report on the successful rendezvous technique of double-balloon endoscopy (DBE) (EI-530B; Fujifilm, Osaka, Japan) combined with the percutaneous SpyGlass ${ }^{\circledR}$ direct visualization system (SDVS) (Boston Scientific Corp., Natick, MA, USA) to treat severe stenosis of a choledochojejunal anastomosis in a patient with pancreaticoduodenectomy. A 71-year-old man with a history of intraductal papillary mucinous neoplasm who had undergone pylorus-preserving pancreaticoduodenectomy 6 months previously was admitted for obstructive jaundice and acute cholangitis. He was referred for endoscopic retrograde cholangiopancreatography (ERCP) using a "short" DBE for internalization of biliary drainage. Locating the choledochojejunal anastomosis was difficult, but was successfully achieved by detecting the ulcer-scar-like lesion and a little biliary flow in the underwater observation ( Video 1). However, the procedure failed because of a severe stenosis consisting of a long stricture with a small orifice in the choledochojejunal anastomosis, which was denoted a type
A2 stricture in the classification of Mönkemüller and Jovanovic [1] and hindered the advancement of several devices. As an alternative, percutaneous transhepatic cholangiodrainage (PTCD) was applied, but this was again precluded by the severe stenosis. As the patient firmly refused to undergo further surgery, DBE-assisted ERCP was reattempted. Indigo carmine was used to mark the location of the choledochojejunal anastomosis and, using a needle knife (KD-V441M; Olympus Medical Systems, Tokyo, Japan), the membranous anastomotic stenosis was incised ( Video 2). When a blind incision was made in the presumed direction of the biliary duct, the cholangiographic image showed leakage of contrast medium into the abdominal cavity ( $\bullet$ Fig. 1 ), indicating that the presumed orientation was incorrect. To assure the correct direction of the incision, the SDVS was inserted via the PTCD route and successfully functioned as a visible light guide, facilitating accurate incision into the biliary duct ( Video 3 ). Consequently, guidewires were advanced through the choledochojejunal anas- tomotic stenosis and dilatation using an 8-mm dilator balloon (QBD-8X3; Cook Medical, Bloomington, IN, USA) was successful, allowing placement of two endoscopic biliary drainage tubes consisting of 5-Fr plastic stents (ZEBD-5-4; Cook Medical) $(\bullet$ Video 4$)$.

A severe stenosis in a choledochojejunal anastomotic site is a significant problem.

\section{Video 1}

The ulcer-scar-like choledochojejunal anastomosis was found, and a little biliary flow was detected in the underwater observation.

\section{Video 2}

The choledochojejunal anastomosis was identified using indigo carmine, and an incision was made with a needle knife.

\section{Video 3}

Incision using the needle knife proceeded toward the light of the SpyGlass ${ }^{\circledR}$ direct visualization system, which was inserted via the percutaneous transhepatic cholangiodrainage route as a guide, and the biliary duct was successfully accessed.

\section{Video 4}

The choledochojejunal anastomosis was dilated using a biliary dilator balloon, and two endoscopic biliary drainage tubes consisting of 5-Fr plastic stents were placed.
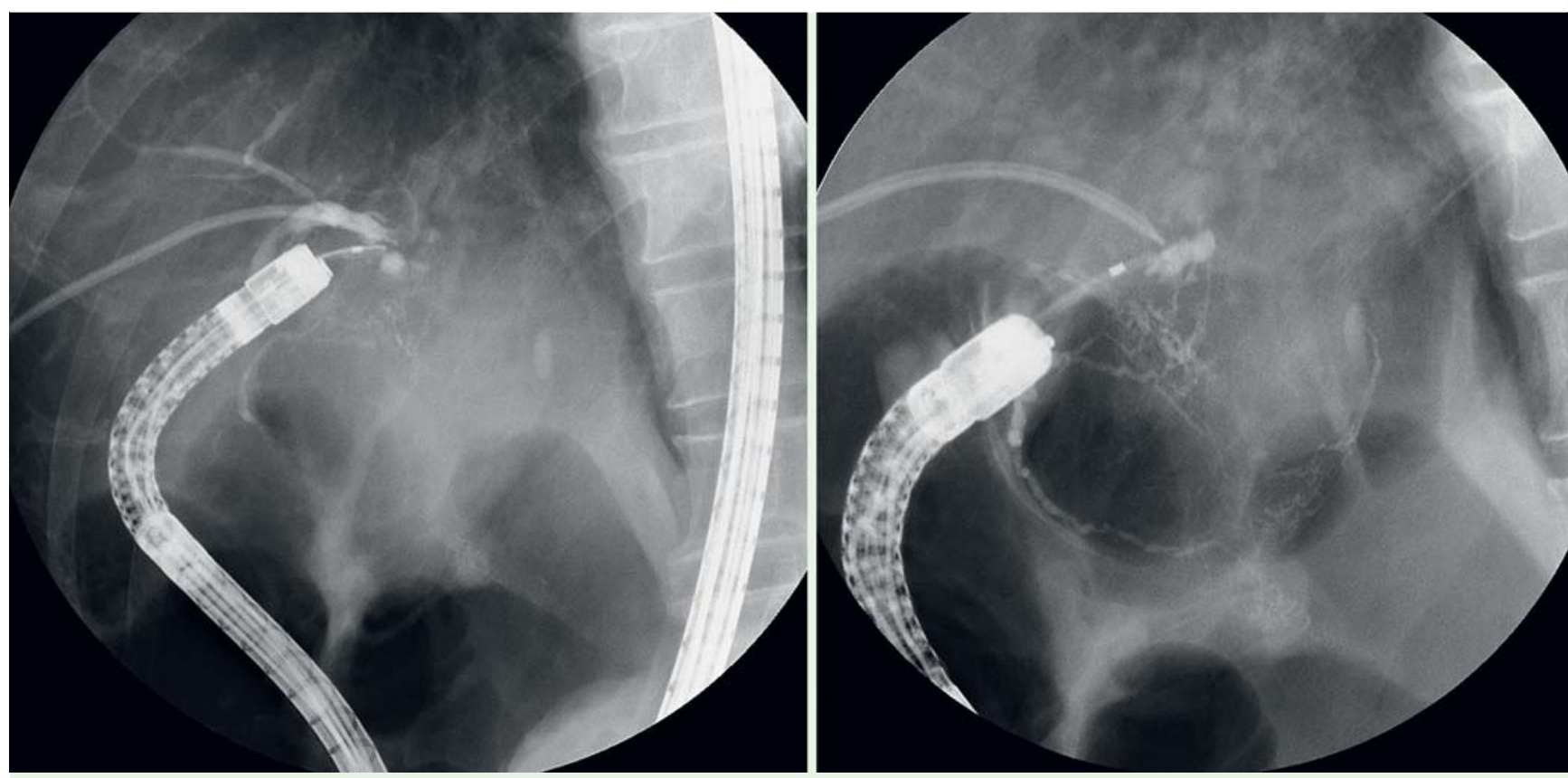

Fig. 1 Cholangiography showed leakage of contrast media into the abdominal cavity after the incision into the choledochojejunal anastomosis. 
DBE-assisted ERCP has already gained acceptance for its less invasive technique and high-quality performance [2,3], and its applications in rendezvous techniques have been reported [4,5]. Adding to these, the rendezvous technique in combination with SDVS reported here will expand the possibilities of endoscopic approaches for extremely difficult cases where DBE-assisted ERCP or PTCD fails to offer patients an alternative that will allow them to avoid surgery.

Endoscopy_UCTN_Code_TTT_1AR_2AC

Competing interests: None

Masaaki Shimatani, Makoto Takaoka, Tsukasa Ikeura, Toshiyuki Mitsuyama, Kota Kato, Kazuichi Okazaki

Third Department of Internal Medicine, Kansai Medical University, Osaka, Japan

\section{References}

1 Mönkemüller K, Jovanovic I. Endoscopic and retrograde cholangiographic appearance of hepaticojejunostomy strictures: a practical classification. World J Gastrointest Endosc 2011; 3: 213-219

2 Shimatani M, Matsushita M, Takaoka M et al. Effective "short" double-balloon enteroscope for diagnostic and therapeutic ERCP in patients with altered gastrointestinal anatomy: a large case series. Endoscopy 2009; 41: 849-854

3 Shimatani M, Takaoka M, Matsushita M et al. Endoscopic approaches for pancreatobiliary diseases in patients with altered gastrointestinal anatomy. Dig Endosc 2014; 26: $70-78$

4 Mönkemüller K, Popa D, McGuire B et al. Double-balloon enteroscopy-ERCP rendezvous technique. Endoscopy 2013; 45: E333-334

5 Itoi T, Ishii K, Sofuni A et al. Single balloon enteroscopy-assisted ERCP using rendezvous technique for sharp angulation of Roux-en-Y limb in a patient with bile duct stones. Diagn Ther Endosc 2009; 2009: 154084

\section{Bibliography}

DoI http://dx.doi.org/ 10.1055/s-0034-1365785 Endoscopy 2014; 46: E275-E276

(c) Georg Thieme Verlag KG Stuttgart · New York ISSN 0013-726X

\section{Corresponding author}

Masaaki Shimatani, MD

Third Department of Internal Medicine Kansai Medical University 2-5-1 Shinmachi, Hirakata Osaka 573-1010

Japan

Fax: +81-72-8042524

shimatam@hirakata.kmu.ac.jp 